\title{
Rupture and Dissection Pulmonary Artery Aneurysm in a patient with a complex Congenital Heart Disease
}

\author{
Santiago Herrero ${ }^{1 *}$ and Luis Rodriguez Redondo ${ }^{2}$ \\ ${ }^{1}$ Department of Cardiac Critical Care, The Jilin Heart Hospital, China \\ ${ }^{2}$ University of Oviedo, Spain
}

*Corresponding author: Santiago Herrero, Department of Cardiac Critical Care, The Jilin Heart Hospital, China.

Keywords: Cardiogenic shock; Pulmonary artery; Aneurysm; Dissection; Congenital heart disease

\section{Introduction}

We present the images of an extremely infrequent case of acute pulmonary artery aneurysm disecction in a patient, male, 38 years old with complex congenital heart disease: transposition of great vessels (TGV) corrected, with subpulmonary ventricular septal defect (VSD) and very severe pulmonary hypertension, not surgically corrected. Atrial fibrillation anticoagulated with warfarin.

\section{Admission}

Suddenly episode of loose prickly chest pain that increased with respiratory movements and radiated to the sternum, throat and ear. Increased jugular venous pressure. Poor perfusion, BP 90/45 torr. Pulmonary auscultation with striking tubal murmur $1 / 3$ inferior of the left lung.

\section{Evolution}

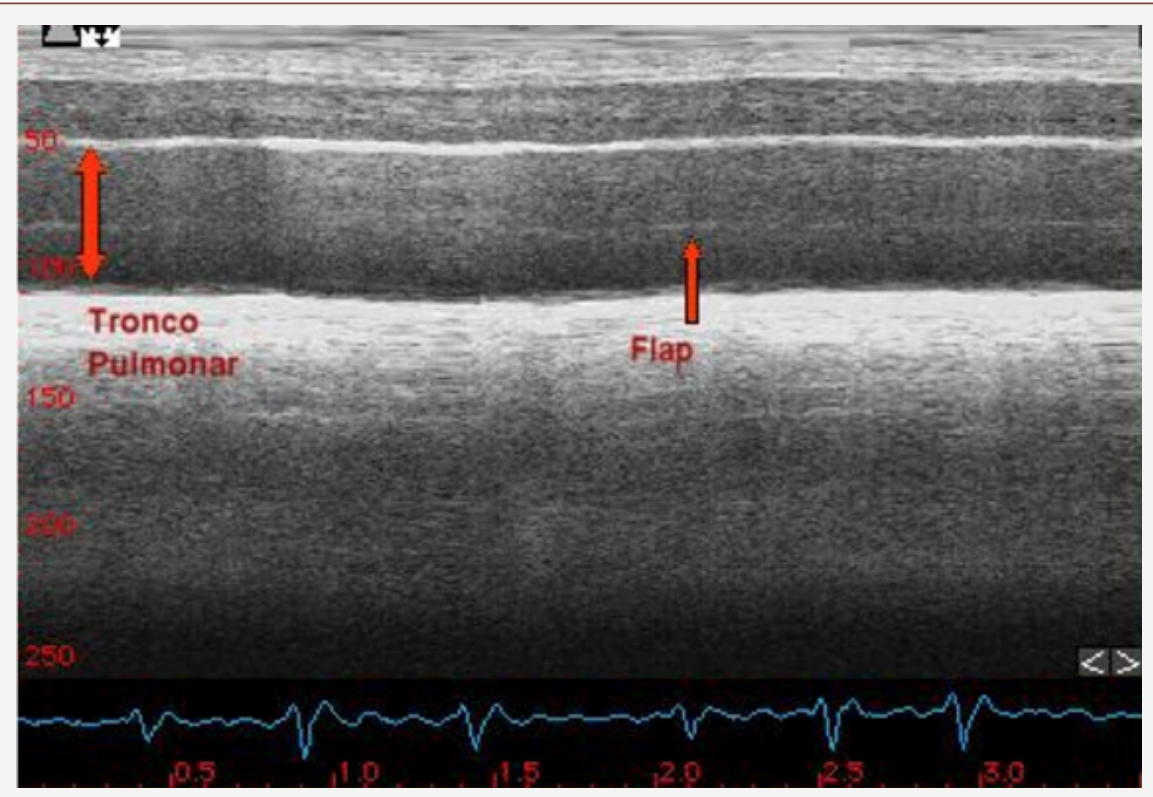

Figure 1 


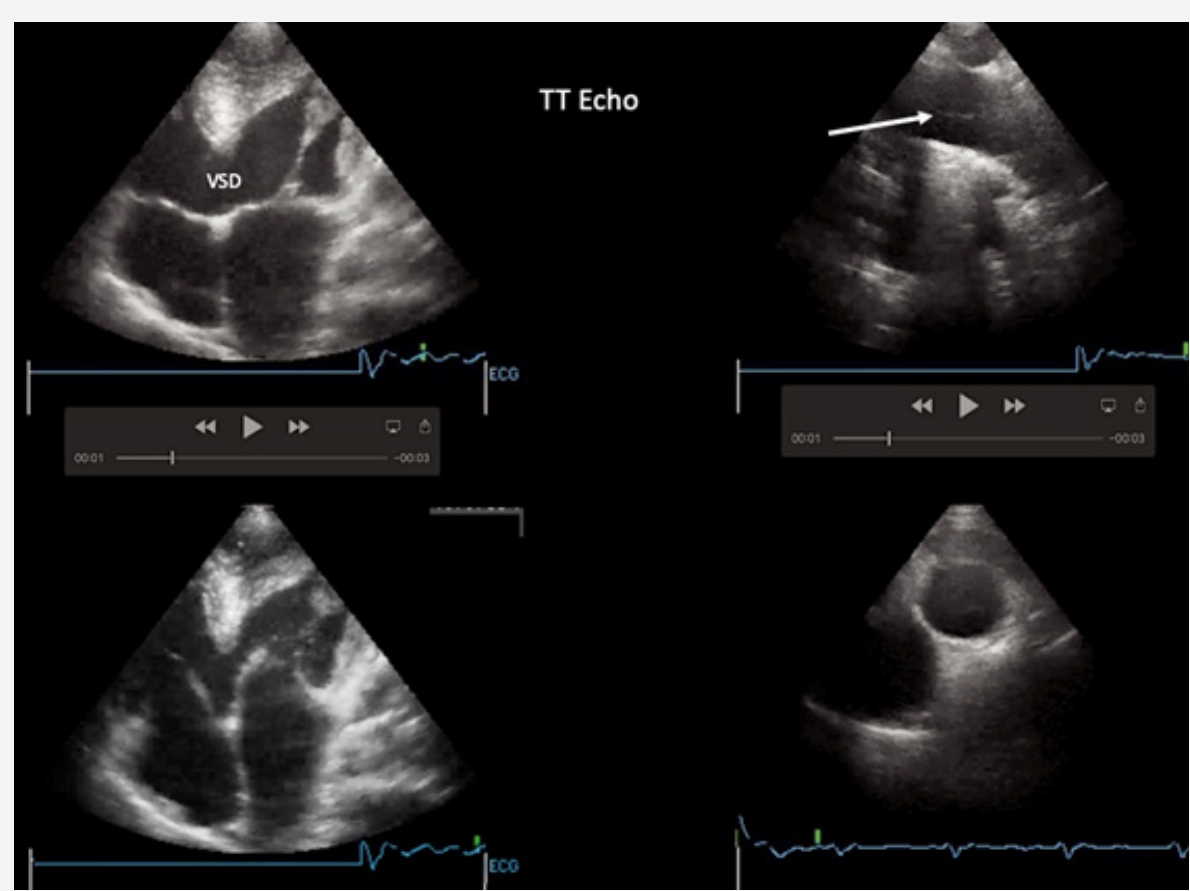

Figure 2
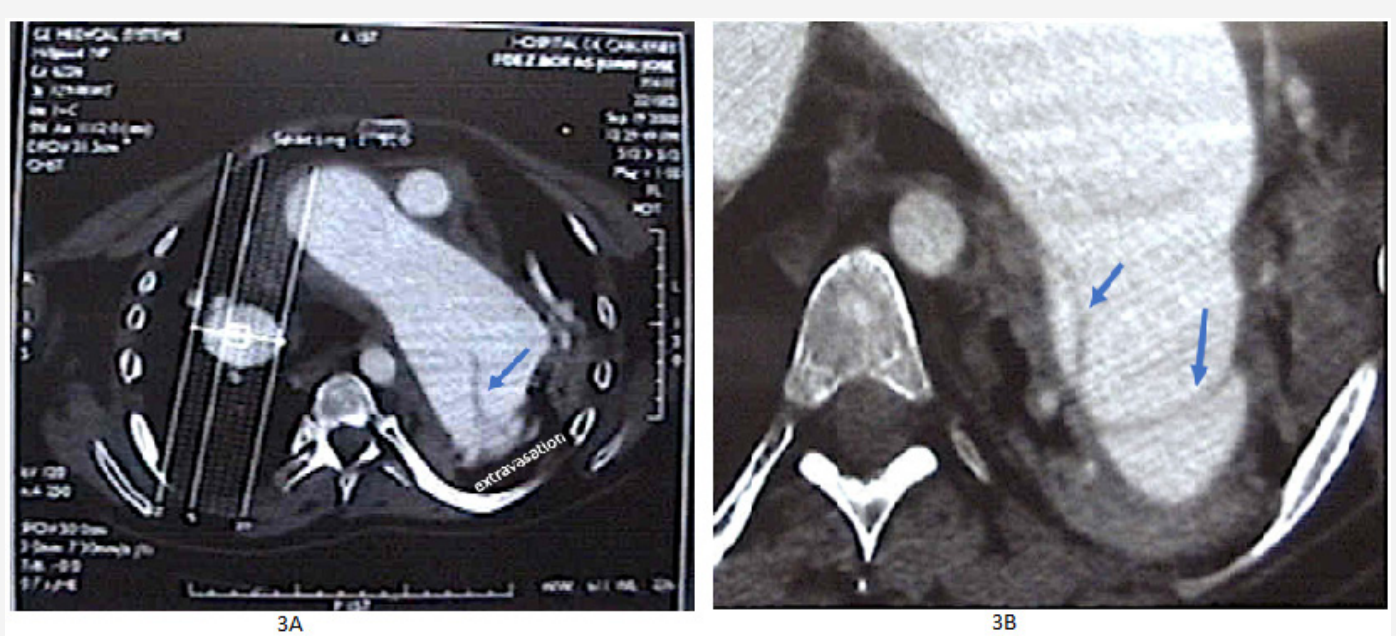

Figure 3

Proposal for combined lung and heart transplantation. Suddenly the patient collapsed and died (Figure 1-3).

\section{Images}

Image 1: TTE (M mode), intimal flap visualized in the pulmonary trunk and the left pulmonary artery.

Image 2: Transthoracic Echocardiography (TTE): Situs solitus corrected TGV + mild-moderate pulmonary stenosis. Large subpulmonary VSD. Intimal flap over pulmonary trunk and left pulmonary artery.

Image 3: Thorax Angio CT: a. Large dilation of the pulmonary artery and its main branches and hyperdense band adjacent to the left inferior interlobar artery secondary to extravasation of blood and contrast

b. Aneurysmal dissection of the left pulmonary artery and its lower left interlobar branch.

\section{Acknowledgement}

None.

\section{Conflict of Interest}

There is no declared competing conflict of interest between authors. 